\title{
ON AN INTEGRAL REPRESENTATION FOR THE GENUS SERIES FOR 2-CELL EMBEDDINGS
}

\author{
D. M. JACKSON
}

\begin{abstract}
An integral representation for the genus series for maps on oriented surfaces is derived from the combinatorial axiomatisation of 2-cell embeddings in orientable surfaces. It is used to derive an explicit expression for the genus series for dipoles. The approach can be extended to vertex-regular maps in general and, in this way, may shed light on the genus series for quadrangulations. The integral representation is used in conjunction with an approach through the group algebra, $\mathbb{C G}_{n}$, of the symmetric group [11] to obtain a factorisation of certain Gaussian integrals.
\end{abstract}

\section{A POWER SERIES REPRESENTATION FOR THE GENUS SERIES}

A map is a 2-cell embedding of a connected unlabelled graph $\mathscr{G}$, with loops and multiple edges allowed, in a closed surface $\Sigma$, without boundary, which is assumed throughout to be oriented. The deletion of $\mathscr{G}$ separates $\Sigma$ into regions homeomorphic to open discs, called the faces of the map, and the number of edges bordering a face is called its degree. A map is rooted by distinguishing a mutually incident vertex, edge and face. The genus series for a class of maps is the formal generating series for the number of inequivalent maps with respect to genus, and the numbers of vertices, edges and faces. It is assumed hereinafter that maps are rooted.

The general approach adopted here combines ideas of Jackson and Visentin [11] with those of 't Hooft [8] and Bessis, Itzykson and Zuber [3] who, in the above terminology, derived the genus series for diagrams akin to a class of maps by techniques from conformal field theory. Although [8] and [3] are important papers, they have remained largely inaccessible to combinatorialists because of their uncertainty about the automorphisms of these diagrams as combinatorial structures.

In this paper, an explicit construction is given for an integral representation for the genus series for general maps directly from the combinatorial axiomatisation for embeddings on oriented surfaces. Moreover, we also develop methods which are extensible to vertex-regular maps (vertices have the same degree) and thence, by restriction, to quadrangulations (maps whose faces are bounded by four edges). This is done by examining dipoles (maps with two vertices) in detail. Although the argument is an algebraic one, based on the ring of formal power series, to assert that particular series belong to the ring, it is necessary to

Received by the editors July 1, 1992 and, in revised form, August 15, 1993.

1991 Mathematics Subject Classification. Primary 05A15, 20C15; Secondary 57N37. 
appeal to the existence of $\int_{\mathbb{R}} e^{-\alpha t^{2}} d t$ for positive real $\alpha$. Thus, for example,

$$
\frac{\int_{\mathbb{R}} e^{-t^{2}+\left(x_{1} t+x_{2} t^{2}+x_{3} t^{3}+\cdots\right)} d t}{\int_{\mathbb{R}} e^{-t^{2}} d t} \in \mathbb{Q}\left[x_{1}, x_{2}, \ldots\right][[t]],
$$

where $x_{1}, x_{2}, \ldots$ are commutative indeterminates. For technical reasons, it is also necessary to appeal to the existence of Haar measure for the unitary group.

The starting point of this investigation is a result, stated below as Theorem 1.1 , giving an explicit power series representation for the genus series. From the point of view of the present discussion, it is important to note that it is proved directly from the combinatorial axiomatisation for embeddings, and the group algebra of the symmetric group. The details of proof are given in [11], to which the reader is referred, and no further appeal to the axiomatisation is necessary here. The following notation is needed for stating the theorem. If $\theta=\left(\theta_{1}, \theta_{2}, \ldots\right)$, with $\theta_{1} \geq \cdots \geq \theta_{k}>\theta_{k+1}=\cdots=0$, then $\theta$ is a partition of $n=\theta_{1}+\cdots+\theta_{k}$, and we write $\theta \vdash n$. The weight of $\theta$ is $|\theta|=n$, and the length of $\theta$ is $l(\theta)=k$. Let $m_{j}(\theta)$ be the number of occurrences of $j$ in $\theta$. We write $\theta=\left[1^{m_{1}(\theta)} 2^{m_{2}(\theta)} \ldots\right]$, suppressing terms for which $m_{j}(\theta)=0$, and let $m(\theta)=\left(m_{1}(\theta), m_{2}(\theta), \ldots\right)$. If $\theta \vdash n$, let $\mathscr{C}_{\theta}$ denote the conjugacy class of $\mathfrak{G}_{n}$ indexed by $\theta$. The number of elements in this class is denoted by $h^{\theta}$. Clearly, $h^{\theta}=(2 n) ! g(\theta)$ where $g^{-1}(\theta)=\prod_{j \geq 0} j^{m_{j}(\theta)} m_{j}(\theta)$ !. For $\pi \in \mathfrak{G}_{n}$, let $\kappa(\pi)$ denote the number of cycles in $\pi$, so $\kappa(\pi)=l(\theta)$ if $\pi \in \mathscr{C}_{\theta}$. If $\mathbf{x}=\left(x_{1}, x_{2}, \ldots\right)$ and $\mathbf{i}=\left(i_{1}, i_{2}, \ldots\right)$ then $\mathbf{x}^{\mathbf{i}}$ denotes $x_{1}^{i_{1}} x_{2}^{i_{2}}, \ldots$, where $x_{1}, x_{2}, \ldots$ are commutative indeterminates. If $f \in \mathbb{R}[[\mathbf{x}]]$, where $\mathbb{R}$ is a ring, then the coefficient of $\mathbf{x}^{\mathbf{i}}$ in $f$ is denoted by $[\mathbf{x}]^{\mathbf{i}} f$. For $f \in \mathbb{Q}[[x, y, z]]$, let $\Omega_{u}$ denote the mapping

$$
\Omega_{u} f(x, y, z)=1+2 u^{2} z \frac{\partial}{\partial z} f\left(x u^{-1}, y u^{-1}, \frac{1}{2} z u\right) .
$$

For a partition $\alpha$, let $\nu$ be an arbitrary but fixed element of $\mathscr{C}_{\alpha}$ and let

$$
A_{\alpha}(y)=\sum_{\pi \in \nu \mathscr{C}_{\left[2^{n}\right]}} y^{\kappa(\pi)}
$$

with the convention that $A_{\alpha}=0$ if $|\alpha| \not \equiv 0 \bmod 2$. This polynomial, which is a class function for $\mathfrak{G}_{2 n}$, is fundamental to the argument. The result may now be stated.

Theorem 1.1. Let $m_{g, \mathbf{i}, j, k}$ be the number of maps of genus $g$ with $j$ faces, $k$ edges and $i_{r}$ vertices of degree $r=0,1,2, \ldots$ and let

$$
M(u, \mathbf{x}, y, z)=\sum_{g, \mathbf{i}, j, k \geq 0} m_{g, \mathbf{i}, j, k} \mathbf{x}^{\mathbf{i}} y^{j} z^{k} u^{g},
$$

the genus series for maps. Then

$$
M\left(u^{2}, \mathbf{x}, y, z\right)=\Omega_{u} \log \sum_{k \geq 0} \frac{2^{k} z^{k}}{(2 k) !} \sum_{\nu \vdash 2 k} h^{\nu} A_{\nu}(y) \mathbf{x}^{m(\nu)} \in \mathbb{Q}[u, \mathbf{x}, y][[z]] .
$$

The coefficient ring for $M$, as a formal power series in $z$, is a polynomial ring because there is only a finite number of maps with a given number of edges.

An integral representation for $A_{\alpha}$ is constructed in $\S 2$ from a discontinuous integral representation for $\delta_{p, q}$, and an integral representation for the genus 
series is then derived from this. Discontinuous integral representations of combinatorial functions arise elsewhere (see, for example, [12] for a representation of $<$ ). Two examples of the use of this representation are given in $\S 3$. In the first, a relationship is obtained between the genus series for smooth maps (no monovalent vertices) and all maps, simply by transforming the integral. A similar result holds for homeomorphically irreducible maps (no bivalent vertices). In the second example, concerning monopoles (maps with one vertex), the integration has to be carried out explicitly. The main part of this section deals with an approach to vertex-regular maps, which is developed by studying dipoles in detail. Section 4 contains two results which may be regarded as applications of the theory of maps. By considering the centre of the group algebra of the symmetric group, it is possible to express certain of the integrals appearing here as character sums. We also obtain a factorisation of integrals of this type, into a product of similar integrals, by considering the following result connecting quadrangulations and maps. Let $M_{4}(u, x, y, z)$ denote the genus series for quadrangulations, and let $M(u, x, y, z)$ denote $M(u, \mathbf{x}, y, z)$ at $x_{1}=x_{2}=\cdots=x$. Then [11]

(2) $M_{4}\left(u^{2}, x, y, z\right)=\frac{1}{2}\left\{M\left(4 u^{2}, x+u, x, z^{2} y\right)+M\left(4 u^{2}, x-u, x, z^{2} y\right)\right\}$.

This may be of significance in the context of matrix models in statistical mechanics and in the context of the $\phi^{4}$ - and Penner models [9]. A direct proof of the factorisation may explain whether (2) can be adjusted to hold for maps on nonorientable surfaces. It should be noted that, in these models, monovalent and bivalent vertices are suppressed and that, in effect, the maps (which are therefore smooth and homeomorphically irreducible) are counted with respect to their genus and number of faces. Under these conditions, this number is of course finite, and we denote the series by $M_{\overline{\{1,2\}}}(u, x, y, z)$. It is $M_{4}(u, 1, y, 1)$ and $M_{\overline{\{1,2\}}}(u, 1, y, 1)$ which are closely related to the perturbation series for the $\phi^{4}$ - and Penner models [9], whose examination in the double scaling limit is currently of interest.

\section{AN INTEGRAL REPRESENTATION FOR THE GENUS SERIES}

We begin by deriving an expression for $A_{\alpha}(N)$ in terms of the function $\psi$ defined by

$$
\psi: \mathbb{N}^{2} \times \mathbb{N}^{2} \rightarrow\{0,1\}:((p, q),(r, s)) \mapsto \delta_{p, s} \delta_{q, r},
$$

where $\mathbb{N}$ is the set of all positive integers.

Lemma 2.1. Let $N$ be a positive integer, and let $\nu$ be a fixed permutation in $\mathscr{C}_{\alpha}$, where $\alpha \vdash 2 n$. Then

$$
A_{\alpha}(N)=\sum_{1 \leq i_{1}, \ldots, i_{2 n} \leq N} \sum_{\rho \in \mathscr{C}_{\left[2^{n}\right]}} \prod_{j=1}^{2 n} \psi\left(\left(i_{j}, i_{\nu(j)}\right),\left(i_{\rho(j)}, i_{\nu \rho(j)}\right)\right) .
$$

Proof. From (1) and the cycle decomposition for permutations,

$$
N^{\kappa(\pi)}=\sum_{1 \leq i_{1}, \ldots, i_{2 n} \leq N} \prod_{j=1}^{2 n} \delta_{i_{j}, i_{\pi(j)}}
$$


so

$$
\sum_{\pi \in \nu \mathscr{C}_{\left[2^{n}\right]}} N^{\kappa(\pi)}=\sum_{1 \leq i_{1}, \ldots, i_{2 n} \leq N} \sum_{\pi \in \nu \mathscr{C}_{\left[2^{n}\right]}} \prod_{j=1}^{2 n} \delta_{i_{j}, i_{\pi(j)}}
$$

But

$$
\sum_{\pi \in \nu \mathscr{C}_{\left[2^{n}\right]}} \prod_{j=1}^{2 n} \delta_{i_{j}, i_{\pi(j)}}=\sum_{\rho \in \mathscr{C}_{\left[2^{n}\right]}} \prod_{j=1}^{2 n} \delta_{i_{j}, i_{\nu \rho(j)}}=\sum_{\rho \in \mathscr{C}_{\left[2^{n}\right]}} \prod_{j=1}^{2 n} \delta_{i_{j}, i_{\nu \rho(j)}} \delta_{i_{\rho(j)}, i_{\nu(j)}}
$$

since $\prod_{j=1}^{2 n} \delta_{i_{j}, i_{\nu \rho(j)}}=\prod_{j=1}^{2 n} \delta_{i_{\rho(j)}, i_{\nu(j)}}=0$ or 1 , for each $\rho \in \mathscr{C}_{\left[2^{n}\right]}$. The result follows from (3).

$A_{\alpha}(y)$, which is required in the determination of the genus series, is obtained by exhibiting $A_{\alpha}(N)$ as an explicit polynomial in $N$, and by then replacing $N$ throughout by the indeterminate $y$. This argument is an algebraic one and is used repeatedly. Further attention will not be drawn to it. In view of this lemma, we call $\psi$ the oriented connector function for maps. Our aims are to determine the sum over involutions and to construct an integral representation for the function $\psi$. These are now addressed in turn.

Definition 2.2. Let $K, r, s, d_{1}, \ldots, d_{N}$, be positive integers, let $q$ be an integer such that $0 \leq q<K$, and let $\mathbf{d}=\left(d_{1}, \ldots, d_{N}\right)$. The linear functional \langle\rangle$_{K, \mathrm{~d}}$ on $\mathbb{Q}\left[\left[x_{1}, \ldots, x_{N}\right]\right]$ is defined by

$$
\begin{aligned}
\left\langle x_{j}^{r} x_{k}^{s}\right\rangle_{K, \mathbf{d}} & =\left\langle x_{j}^{r}\right\rangle_{K, \mathbf{d}} \cdot\left\langle x_{k}^{s}\right\rangle_{K, \mathbf{d}} \quad \text { if } j \neq k, \\
\left\langle x_{j}^{K n+q}\right\rangle_{K, \mathbf{d}} & =d_{j}^{-n} h^{\left[K^{n}\right]} \delta_{q, 0} .
\end{aligned}
$$

Since $\left\langle x_{j} x_{k}\right\rangle_{2, \mathrm{~d}}=d_{j}^{-1} \delta_{j k}$, an integral representation for the connector function can be constructed systematically by generalising an integral representation of this functional from a set of singly indexed indeterminates to a set of doubly indexed indeterminates. Because of the importance of fixed point free involutions in the analysis of maps, attention is confined here to the case $K=2$, although an analogous result holds for $K>2$.

Let $1 \leq i_{1}, \ldots, i_{2 n} \leq N$, be integers, and let $z_{p, q}$ for $1 \leq p, q \leq N$ be indeterminates with $z_{p, q}=z_{q, p}$. Clearly, $\prod_{j=1}^{2 n} z_{i_{j}, i_{\rho(j)}}=t^{2}$ where $t$ is a monomial in the set of indeterminates and $\rho \in \mathscr{C}_{\left[2^{n}\right]}$, so we may write $\prod_{j=1}^{2 n} z_{i_{j}, i_{\rho(j)}}^{1 / 2}=t$. A canonical construction for $t$ as a product of $z_{p, q}$ over $n$ pairs $(p, q)$ can be given. However, it is more convenient to work with the product over $1, \ldots, 2 n$, having recognised that the square root is simply a syntactic device.

The following is a summation theorem for the linear functional \langle\rangle$_{2, d}$. It is an alternative formulation of Wick's lemma [3], but is in a form which is extensible to conjugacy classes other than $\mathscr{C}_{\left[K^{N}\right]}$, as shown in $\S 5$.

Lemma 2.3. Let $i_{1}, \ldots, i_{2 n} \leq N$ be positive integers, let $\mathbf{x}=\left(x_{1}, \ldots, x_{N}\right)$ and let $g_{k}(\mathbf{x})$ be a homogeneous linear polynomial in $\mathbf{x}$ for $k=1, \ldots, N$. Then

$$
\sum_{\rho \in \mathscr{E}_{\left.2^{n}\right]}} \prod_{j=1}^{2 n}\left\langle g_{i}(\mathbf{x}) g_{i_{\rho(j)}}(\mathbf{x})\right\rangle_{2, \mathbf{d}}^{1 / 2}=\left\langle g_{i_{1}}(\mathbf{x}) \cdots g_{i_{2 n}}(\mathbf{x})\right\rangle_{2, \mathbf{d}}
$$


Proof. We first prove that

$$
\sum_{\rho \in \mathscr{C}_{\left[2^{n}\right]}} \prod_{j=1}^{2 n}\left\langle x_{i_{j}} x_{i_{\rho(j)}}\right\rangle_{2, \mathbf{d}}^{1 / 2}=\left\langle x_{i_{1}} \cdots x_{i_{2 n}}\right\rangle_{2, \mathrm{~d}} .
$$

Let $\left(i_{1}, \ldots, i_{2 n}\right)=\left[1^{a_{1}} 2^{a_{2}} \cdots N^{a_{N}}\right]$. There are two cases to be considered.

Case 1. If one of the $a_{i}$ 's is odd, suppose it to be $a_{k}$. Now $\rho(j) \neq j$ for $\rho \in \mathscr{C}_{\left[2^{n}\right]}$ so there is some $\left(i_{j}, i_{\rho(j)}\right)$ such that $i_{\rho(j)} \neq i_{j}$. Thus

$$
\prod_{j=1}^{2 n}\left\langle x_{i_{j}} x_{i_{\rho(j)}}\right\rangle_{2, \mathrm{~d}}^{1 / 2}=\left(d_{i_{1}} \cdots d_{i_{2 n}}\right)^{-1 / 2} \prod_{j=1}^{2 n} \delta_{i_{j}, i_{\rho(j)}}=0
$$

from Definition 2.2, so the left-hand side is zero. But $\left\langle x_{i_{1}} \cdots x_{i_{2 n}}\right\rangle_{2, \mathrm{~d}}=$ $\left\langle x_{1}^{a_{1}}\right\rangle_{2, \mathrm{~d}} \cdots\left\langle x_{N}^{a_{N}}\right\rangle_{2, \mathrm{~d}}$ so the right-hand side is zero, from Definition 2.2. Thus (4) holds in this case.

Case 2. Suppose next that none of the $a_{j}$ 's is zero, so $a_{j}=2 k_{j}$ for $j=$ $1, \ldots, N$. Then

$$
\begin{aligned}
& \sum_{\rho \in \mathscr{C}_{\left[2^{n}\right]}} \prod_{j=1}^{2 n}\left\langle x_{i_{j}} x_{i_{\rho(j)}}\right\rangle_{2, \mathbf{d}}^{1 / 2}=\sum_{\rho \in \mathscr{C}_{\left[2^{n}\right]}}\left(d_{i_{1}} \cdots d_{i_{2 n}}\right)^{-1 / 2} \prod_{j=1}^{2 n} \delta_{i_{j}, i_{\rho(j)}} \\
& \quad=\left(d_{1}^{k_{1}} \cdots d_{N}^{k_{N}}\right)^{-1} \prod_{r=1}^{N} \sum_{\rho \in \mathscr{C}_{\left[2^{n}\right]}} 1=\prod_{r=1}^{N} d_{r}^{-k_{r}} h^{\left[2^{k_{r}}\right]}=\left\langle x_{1}^{a_{1}}\right\rangle_{2, \mathbf{d}} \cdots\left\langle x_{N}^{a_{n}}\right\rangle_{2, \mathbf{d}},
\end{aligned}
$$

from Definition 2.2, so (4) also holds in this case, and therefore in general.

Now let $g_{r}(\mathbf{x})=\sum_{k=1}^{N} a_{r k} x_{k}$. Then, from (4),

$$
\begin{aligned}
& \left\langle g_{i_{1}}(\mathbf{x}) \cdots g_{i_{2 n}}(\mathbf{x})\right\rangle_{2, \mathbf{d}}=\sum_{q_{1}, \ldots, q_{2 n}=1} a_{i_{1} q_{1}} \cdots a_{i_{2 n} q_{2 n}}\left\langle x_{q_{1}} \cdots x_{q_{2 n}}\right\rangle_{2, \mathbf{d}} \\
& =\sum_{\rho \in \mathscr{C}_{\left[2^{n}\right]}} \sum_{q_{1}, \ldots, q_{2 n}=1}^{N} a_{i_{1} q_{1}} \cdots a_{i_{2 n} q_{2 n}}\left\langle x_{q_{1}} x_{q_{\rho(1)}}\right\rangle_{2, \mathbf{d}}^{1 / 2} \cdots\left\langle x_{q_{2 n}} x_{q_{\rho(2 n)}}\right\rangle_{2, \mathbf{d}}^{1 / 2}
\end{aligned}
$$

which gives the result.

An integral representation for the connector function can be constructed systematically by generalising an integral representation of the linear functional (Definition 2.2) from a set of singly indexed indeterminates to a set of doubly indexed indeterminates. We begin by deriving an integral representation for \langle\rangle$_{2, \mathbf{d}}$ from $\left\langle x^{n}\right\rangle_{2, \mathbf{d}}$.

Lemma 2.4. Let $d_{1}, \ldots, d_{N}$ be nonnegative integers, let $\mathbf{x}=\left(x_{1}, \ldots, x_{N}\right)$ and let $f(\mathbf{x})$ be a polynomial in $x_{1}, \ldots, x_{N}$. Then

$$
\langle f(\mathbf{x})\rangle_{2, \mathbf{d}}=\frac{\int_{\mathbf{R}^{N}} f(\mathbf{x}) e^{-\frac{1}{2}\left(d_{1} x_{1}^{2}+\cdots+d_{N} x_{N}^{2}\right)} d \mathbf{x}}{\int_{\mathbb{R}^{N}} e^{-\frac{1}{2}\left(d_{1} x_{1}^{2}+\cdots+d_{N} x_{N}^{2}\right)} d \mathbf{x}} .
$$

Proof. It is sufficient to prove the result for the monomial $x_{1}^{i_{1}} \cdots x_{N}^{i_{N}}$, where $i_{1}, \ldots, i_{N}$ are nonnegative integers. Let $I_{k}(\alpha)=\int_{\mathbb{R}} x^{k} e^{-\alpha x^{2}} d x$ where $\alpha$ is real and nonnegative to assure convergence of the integral. Then

$$
I_{2 k-1}(\alpha)=0, \quad \text { and } \quad I_{2 k}(\alpha)=\frac{(2 k) !}{k ! 4^{k}} \frac{\sqrt{\pi}}{\alpha^{k+1 / 2}} .
$$


It is convenient to normalise this and use $I_{k}^{\prime}(\alpha)=I_{k}(\alpha) / I_{0}(\alpha)$. Then

$$
I_{2 k}^{\prime}(\alpha)=\frac{1}{(2 \alpha)^{k}} h^{\left[2^{k}\right]} \in \mathbb{Q} \text {. }
$$

Thus, from Definition 2.2, $\left\langle x_{j}^{n}\right\rangle_{2, \mathrm{~d}}=I_{n}^{\prime}\left(\frac{1}{2} d_{j}\right)$ so, again from Definition 2.2,

$$
\left\langle x_{1}^{i_{1}} \cdots x_{N}^{i_{N}}\right\rangle_{2, \mathrm{~d}}=I_{i_{1}}^{\prime}\left(\frac{1}{2} f_{1}\right) \cdots I_{i_{N}}^{\prime}\left(\frac{1}{2} d_{N}\right)=\prod_{j=1}^{N} \frac{\int_{\mathbb{R}} x_{j}^{i_{j}} e^{-\frac{1}{2} d_{j} x_{j}^{2}} d x_{j}}{\int_{\mathbb{R}} e^{-\frac{1}{2} d_{j} x_{j}^{2}} d x_{j}}
$$

so the result holds in this case and therefore in general, by linearity.

The multiplicative property of \langle\rangle$_{2, d}$ is seen to be a consequence of the multiplicativity of the kernel of the integral. To construct an integral representation of $\psi$, it is necessary to replace $x_{1}, \ldots, x_{N}$ by a doubly indexed set of indeterminates $a_{11}, a_{12}, \ldots, a_{N N}$. Clearly $\left\langle a_{p q} a_{r s}\right\rangle_{2, \mathbf{I}_{N}}=\delta_{p, r} \delta_{q, s}$, where $\mathbf{I}_{N}$ is the $N \times N$ identity matrix, but this is not the desired evaluation of $\psi$. In the next result, an explicit construction is given for deriving the correct one.

\section{Corollary 2.5.}

$$
\psi((p, q),(r, s))=\left\langle m_{p q} m_{r s}\right\rangle_{2, \Delta},
$$

where $[\Delta]_{j k}=2-\delta_{j k}$ and $\left[m_{j k}\right]$ is an $N \times N$ Hermitian complex matrix.

Proof. Let $d_{j k}$ be nonnegative reals and $a_{j k}$ be indeterminates for $1 \leq j$, $k \leq N$. Let $\mathbf{A}=\left[a_{j k}\right]$ and $\mathbf{D}=\left[d_{j k}\right]$ be $N \times N$ matrices. Let $f$ be a polynomial in the elements of $\mathbf{A}$. Then, from Lemma 2.4

$$
\langle f(\mathbf{A})\rangle_{2, \mathbf{D}}=\frac{\int_{\mathbb{R}^{N^{2}}} f(\mathbf{A}) e^{-\frac{1}{2} \sum_{j, k=1}^{N} d_{j k} a_{j k}^{2}} \prod_{j, k=1}^{N} d a_{j k}}{\int_{\mathbb{R}^{N^{2}}} e^{-\frac{1}{2} \sum_{j, k=1}^{N} d_{j k} a_{j k}^{2} \prod_{j, k=1}^{N} d a_{j k}}} .
$$

We determine $f_{p, q}$ and a matrix $\Delta$, explicitly, such that

$$
\left\langle f_{p, q}(\mathbf{A}) f_{r, s}(\mathbf{A})\right\rangle_{2, \Delta}=\delta_{p, s} \delta_{q, r},
$$

since the latter is equal to $\psi((p, q),(r, s))$. Let $m_{j k}=\xi_{j k}\left(a_{j k}+\alpha_{j k} a_{k j}\right)=$ $f_{j, k}(\mathbf{A})$ where $\left\langle a_{p q} a_{r s}\right\rangle_{2, \mathbf{D}}=d_{p q}^{-1} \delta_{p r} \delta_{q s}$, for some $\alpha_{j k} \in \mathbb{C}$ to be determined. Now

$$
\left\langle m_{p q} m_{r s}\right\rangle_{2, \mathbf{D}}=\xi_{p q} \xi_{r s}\left(\left(1+\alpha_{p q} \alpha_{r s}\right) d_{p q}^{-1} \delta_{p r} \delta_{q s}+\left(\alpha_{r s}+\alpha_{p q}\right) d_{p q}^{-1} \delta_{p s} \delta_{q r}\right) .
$$

Thus

$$
\begin{aligned}
1+\alpha_{p q} \alpha_{r s}=0 & \text { for all } p, q, r, s, \\
d_{p q}^{-1} \xi_{p q} \xi_{r s}\left(\alpha_{r s}+\alpha_{p q}\right)=1 & \text { when } p=s, q=r .
\end{aligned}
$$

From (8), $\alpha_{p q}=\alpha_{r s}=c$, where $c$ is a constant. Thus $c= \pm i$. Let $c=i$ so $\alpha_{p q}=i$. Then from (9), $2 i \xi_{p q} \xi_{q p}=d_{p q}$. Let $d_{p q}=2$ when $p \neq q$. Then $i \xi_{p q} \xi_{q p}=1$. Let $\xi_{p q}=1$ if $p<q$, and $\xi_{p q}=-i$ if $p>q$. Moreover, from (9), $2 i d_{p p}^{-1} \xi_{p p}^{2}=1$, so $\xi_{p p}= \pm(1+i)^{-1} d_{p p}^{1 / 2}$. Let $d_{p p}=1$, and select the positive root. Then $\xi_{j j}=(1+i)^{-1}$. It follows that

$$
f_{j, k}(\mathbf{A})= \begin{cases}m_{j k}=a_{j k}+i a_{k j} & \text { if } j<k, \\ m_{j j}=a_{j j} & \text { if } j=k, \\ m_{j k}=a_{k j}-i a_{j k} & \text { if } j>k\end{cases}
$$


Let $\mathbf{M}=\left[m_{j k}\right]_{N \times N}$. Then $\mathbf{M}=\mathbf{M}^{*}$, so $\mathbf{M}$ is Hermitian complex, from (10). Let

$$
[\Delta]_{j k}=d_{j k}=2-\delta_{j k}
$$

This completes the determination of $f_{j k}$ and $\Delta$ so that (7) holds, and the result follows.

A more compact form for $\langle g(\mathbf{M})\rangle_{2, \Delta}$, where $\mathbf{M}$ is an $N \times N$ Hermitian complex matrix, is obtained from (10) and (11) by noting that

$$
\sum_{j, k=1}^{N} d_{j k} a_{j k}^{2}=\sum_{j=1}^{N} a_{j j}-2 \sum_{j \neq k} a_{j k}=\operatorname{tr} \mathbf{M}^{2}
$$

Thus

$$
\int_{\mathbb{R}^{N^{2}}} g(\mathbf{A}) e^{-\frac{1}{2} \sum_{j, k=1}^{N} d_{j k} a_{j k}^{2}} \prod_{1 \leq j, k \leq N} d a_{j k}=\int_{\mathbb{R}^{N^{2}}} g(\mathbf{A}) e^{-\frac{1}{2} \operatorname{tr} M^{2}} \prod_{1 \leq j, k \leq N} d a_{j k}
$$

where $m_{j k}=f_{j k}(\mathbf{A})$, from (10). The integral on the right is denoted by

$$
\int_{\mathscr{V}_{N}} \hat{g}(\mathbf{M}) e^{-\frac{1}{2} \operatorname{tr} \mathbf{M}^{2}} d \mathbf{M}
$$

where $\hat{g}(\mathbf{M})=g(\mathbf{A})$ and $\mathscr{V}_{N}$ is the vector space of $N \times N$ Hermitian complex matrices, which has dimension $N^{2}$ over $\mathbb{R}$. Thus $\left\{a_{j k}: 1 \leq j, k \leq N\right\}$ is a maximal coordinatising set for $\mathscr{V}_{N}$ so, from Lemma 2.4 ,

$$
\langle g(\mathbf{M})\rangle=\frac{\int_{\mathscr{V}_{N}} g(\mathbf{M}) e^{-\frac{1}{2} \operatorname{tr} \mathbf{M}^{2}} d \mathbf{M}}{\int_{\mathscr{V}_{N}} e^{-\frac{1}{2} \operatorname{tr} \mathbf{M}^{2}} d \mathbf{M}}
$$

where, here and throughout, $\langle g(\mathbf{M})\rangle_{2, \Delta}$ is denoted by $\langle g(\mathbf{M})\rangle$, or by $\langle g(\mathbf{M})\rangle_{\mathscr{V}_{N}}$ when explicit reference to the size of the matrices is necessary. We may therefore think of $d \mathbf{M}$ as Lebesgue measure. In particular, from (5),

$$
\int_{\mathscr{V}_{N}} e^{-\frac{1}{2} \operatorname{tr} \mathbf{M}^{2}} d \mathbf{M}=\int_{\mathbb{R}} e^{\left\{-\frac{1}{2} \sum_{j=1}^{N} a_{j j}^{2}-\sum_{j \neq k} a_{j k}^{2}\right\}} \prod_{1 \leq j, k \leq N} d a_{j k}=\left(2^{N} \pi^{N^{2}}\right)^{1 / 2}
$$

An integral representation for $A_{\alpha}(N)$ can now be given by applying Lemma 2.3 in the case of a doubly indexed set of indeterminates.

Theorem 2.6. Let $\alpha=\left[1^{a_{1}} 2^{a_{2}} \cdots\right]$. Let $M\left(u^{2}, x, y, z\right)$ denote $M\left(u^{2}, \mathbf{x}, y, z\right)$ when $x_{1}=x_{2}=\cdots=x_{N}=x$. Then

$$
\begin{aligned}
& \text { (1) } A_{\alpha}(N)=\left\langle\prod_{j \geq 1}\left(\operatorname{tr} \mathbf{M}^{j}\right)^{a_{j}}\right\rangle, \\
& \text { (2) } M\left(u^{2}, \mathbf{x}, N, z\right)=\Omega_{u} \log \left\langle e^{\sum_{k \geq 1} k^{-1} x_{k} \sqrt{2 z}^{k} \operatorname{tr} \mathbf{M}^{k}}\right\rangle, \\
& \text { (3) } M\left(u^{2}, x, N, z\right)=\Omega_{u} \log \left\langle\operatorname{det}\left(\mathbf{I}_{n}-\sqrt{2 z} \mathbf{M}\right)^{-x}\right\rangle .
\end{aligned}
$$

Proof. From Lemma 2.3, after reindexing the variables

$$
\sum_{\rho \in \mathscr{C}_{\ell^{n}}{ }^{n}} \prod_{j=1}^{2 n}\left\langle g_{i}(\mathbf{A}) g_{i_{\rho(j)}}(\mathbf{A})\right\rangle_{2, \mathbf{D}}^{1 / 2}=\left\langle g_{i_{1}}(\mathbf{A}) \cdots g_{i_{2 n}}(\mathbf{A})\right\rangle_{2, \mathbf{D}}
$$


Let $g_{i_{j}}(\mathbf{A})=f_{i_{j}, k_{j}}(\mathbf{A})=m_{i_{j}, k_{j}}$, where $f_{j, k}$ is given by (10). Then

$$
\sum_{\rho \in \mathscr{E}_{\left[2^{n}\right]}} \prod_{j=1}^{2 n}\left\langle m_{i_{j} k_{j}} m_{i_{\rho(j)} k_{\rho(j)}}\right\rangle^{1 / 2}=\left\langle m_{i_{1} k_{1}} \cdots m_{i_{2 n} k_{2 n}}\right\rangle
$$

so, from Corollary 2.5 and (3),

$$
\sum_{\rho \in \mathscr{E}_{\left[2^{n}\right]}} \prod_{j=1}^{2 n} \psi\left(\left(i_{j}, k_{j}\right),\left(i_{\rho(j)}, k_{\rho(j)}\right)\right)=\left\langle m_{i_{1} k_{1}} \cdots m_{i_{2 n} k_{2 n}}\right\rangle
$$

having noted from $(3)$ that $\sqrt{\psi}=\psi$. Let $k_{j}=i_{\nu(j)}$ for $j=i, \ldots, 2 n$. Thus from Lemma 2.1

$$
A_{\alpha}(N)=\sum_{1 \leq i_{1}, \ldots, i_{2 n} \leq N}\left\langle m_{i_{1} i_{\nu(1)}} \cdots m_{i_{2 n} i_{\nu(2 n)}}\right\rangle
$$

and (1) follows. From Theorem 1.1 and (1)

$$
M\left(u^{2}, \mathbf{x}, N, z\right)=\Omega_{u} \log \left(\sum_{\nu} \sqrt{2 z}^{|\nu|} g(\nu)\left\langle\prod_{j \geq 1}\left(x_{j} \operatorname{tr} \mathbf{M}^{j}\right)^{m_{j}(\nu)}\right\rangle\right)
$$

and (2) follows. From (2)

$$
\sum_{k \geq 1} k^{-1} x_{k} \sqrt{2 z}^{k} \operatorname{tr} \mathbf{M}^{k}=x \operatorname{tr} \log \left(\mathbf{I}_{N}-\sqrt{2 z} \mathbf{M}\right)^{-1}=\log \operatorname{det}\left(\mathbf{I}_{N}-\sqrt{2 z} \mathbf{M}\right)^{-x}
$$

by Jacobi's theorem, giving the result.

Jacobi's theorem can be proved in a power series ring (see, for example, [5]). The expression for $M\left(u^{2}, \mathbf{x}, N, z\right)$ can be reduced by diagonalisation from an integral over $\mathbb{R}^{N^{2}}$ to an integral over $\mathbb{R}^{N}$. To simplify integrations, the variables are changed so that the measure is $e^{-x^{2}} d x$, rather than $e^{-x^{2} / 2} d x$ which arises combinatorially.

Corollary 2.7. Let $\alpha \vdash 2 n$, let $p_{\alpha}$ denote $p_{\alpha}\left(\lambda_{1}, \ldots, \lambda_{N}\right)$ and let $d \lambda=d \lambda_{1} \ldots$ $d \lambda_{N}$ where $N$ is a positive integer and let

$$
c_{N, n}=\frac{\sqrt{2}^{N^{2}-N} 2^{n}}{\sqrt{\pi}^{N} \prod_{j=1}^{N} j !} .
$$

Then

(1) $A_{\alpha}(N)=c_{N, n} \int_{\mathbb{R}^{N}} V^{2}(\lambda) e^{-p_{2}} p_{\alpha} d \lambda$,

(2) $M\left(u^{2}, \mathbf{x}, N, z\right)=\Omega_{u} \log \left(c_{N, 0} \int_{\mathbb{R}^{N}} V^{2}(\lambda) e^{-p_{2}+\sum_{k \geq 1} \frac{1}{k} \sqrt{2 z}^{k} x_{k} p_{k}} d \lambda\right)$.

Proof. From Theorem 2.6 and (13)

$$
A_{\alpha}(N)=\frac{1}{\left(2^{N} \pi^{N^{2}}\right)^{1 / 2}} \int_{\mathscr{V}_{N}} \prod_{k \geq 1}\left(\operatorname{tr} \mathbf{M}^{k}\right)^{a_{k}} e^{-\frac{1}{2} \operatorname{tr} \mathbf{M}^{2}} d \mathbf{M}
$$


Now if $f$ is a real valued polynomial on $\mathscr{V}_{N}$, invariant under the adjoint action of the unitary group, then (Theorem $7.4 \mathrm{c}$ of [26])

$$
\int_{\mathscr{V}_{N}} f(\mathbf{M}) d \mathbf{M}=\frac{\pi^{\frac{1}{2} N(N-1)}}{\prod_{j=1}^{N} j !} \int_{\mathbb{R}^{N}} V^{2}(\lambda) f(\Lambda) d \lambda
$$

where $\Lambda=\operatorname{diag}\left(\lambda_{1}, \ldots, \lambda_{N}\right)$. But the integrand is invariant under the adjoint action of unitary group and (1) follows from (14), having replaced $\lambda_{j}$ by $\sqrt{2} \lambda_{j}$. The second part follows from Theorem 2.6 and (14), by the same argument.

The constant factor on the right-hand side of (14) is the volume of the unitary group, and the existence of Haar measure for the unitary group has been assumed. By a theorem of Frobenius, the only finite dimensional real division algebras are $\mathbb{R}, \mathbb{C}, \mathbb{H}$, and there are generalisations of (14) for $\mathbb{R}$ and $\mathbb{H}$. For $\mathbb{R}$, the result is due to Dyson [4].

\section{SMOOTH MAPS, MONOPOLES AND DIPOLES}

A relationship between the genus of maps and smooth maps can be obtained from the integral representation, simply by transforming the integral, with no explicit integration. Since the genus is recoverable from the Euler-Poincare theorem, let $u=1$.

Theorem 3.1. Let $M_{\{\overline{1}\}}(1, x, y, z)$ denote the genus series for smooth maps. Then

$$
\left(v z^{-1} \frac{\partial z}{\partial v}\right) M_{\{\overline{1}\}}(1, x, y, z)+x^{2} y z=M(1, x, y, v)
$$

where $z=z(v)$ is the unique solution of the functional equation $z=v(1+x z)^{2}$. Proof. From Theorem 2.6, with $x_{1}=0, x_{2}=x_{3}=\cdots=x$, we have

$$
M_{\{\overline{1}\}}(1, x, N, z)=2 z \frac{\partial}{\partial z} \log \left\langle e^{x \sqrt{z} \operatorname{tr} \mathbf{M}}\left|\mathbf{I}_{N}-\sqrt{z} \mathbf{M}\right|^{-x}\right\rangle .
$$

But

$$
\left\langle e^{x \sqrt{z} \operatorname{tr} \mathbf{M}}\left|\mathbf{I}_{N}-\sqrt{z} \mathbf{M}\right|^{-x}\right\rangle=K_{N}^{-1} \int_{\mathscr{V}_{N}} e^{-\frac{1}{2} \operatorname{tr} \mathbf{M}^{2}+x \sqrt{z} \operatorname{tr} \mathbf{M}}\left|\mathbf{I}_{N}-\sqrt{z} \mathbf{M}\right|^{-1} d \mathbf{M},
$$

where $K_{N}=\int_{\mathscr{V}_{N}} e^{-\frac{1}{2} \operatorname{tr} \mathbf{M}^{2}} d \mathbf{M}$. But $-\frac{1}{2} \operatorname{tr} \mathbf{M}^{2}+x \sqrt{z} \operatorname{tr} \mathbf{M}=-\frac{1}{2} \operatorname{tr} \mathbf{Q}^{2}+\frac{1}{2} x^{2} z N$ where $\mathbf{Q}=\mathbf{M}-x \sqrt{z} \mathbf{I}_{N}$. Then

$$
\begin{aligned}
\left\langle e^{x \sqrt{z} \operatorname{tr} \mathbf{M}}\left|\mathbf{I}_{N}-\sqrt{z} \mathbf{M}\right|^{-x}\right\rangle & =\frac{e^{\frac{1}{2} x^{2} z N}}{K_{N}} \int_{\mathscr{V}_{N}} e^{-\frac{1}{2} \operatorname{tr} \mathbf{Q}^{2}}\left|(1+x z) \mathbf{I}_{N}-\sqrt{z} \mathbf{Q}\right|^{-x} d \mathbf{Q} \\
& =\left(\frac{e^{1 / 2 x^{2} z}}{(1+x z)^{x}}\right)^{N}\left\langle\operatorname{det}\left(\mathbf{I}_{N}-\frac{\sqrt{z}}{1+x z} \mathbf{Q}\right)^{-x}\right\rangle .
\end{aligned}
$$

The functional equation $v=z(1+x z)^{-2}$ uniquely defines the series $z(v) \in$ $\mathbb{Q}[x][[v]]$. Thus

$$
M_{\{\overline{1}\}}(1, x, N, z)=2 z \frac{\partial}{\partial z} \log \left(\frac{e^{\frac{1}{2} x^{2} z}}{(1+x z)^{x}}\right)^{N}+2 z \frac{\partial}{\partial z} \log \left\langle\left|\mathbf{I}_{N}-v \mathbf{Q}\right|^{-x}\right\rangle
$$


so

$$
v z^{-1} \frac{\partial z}{\partial v} M_{\{\overline{1}\}}(1, x, N, z)-2 v \frac{\partial}{\partial v} \log \left(\frac{e^{\frac{1}{2} x^{2} z}}{(1+x z)^{x}}\right)^{N}=M(1, x, N, z) .
$$

It is a straightforward matter to see from the functional equation for $z$ that

$$
2 v \frac{\partial}{\partial v} \log \left(\frac{e^{\frac{1}{2} x^{2} z}}{(1+x z)^{x}}\right)^{N}=-N z x^{2}
$$

a polynomial in $N$, and the result now follows by replacing $N$ formally by $y$.

The functional equation $z=v(1+x z)^{2}$ identifies $z(v)$ as the generating series for planted plane trees, and it is then a straightforward matter to give Theorem 3.1 a combinatorial proof. This proof amounts to the assertion that the set of all maps with more than one face can be constructed uniquely from a unique smooth map by attaching a plane planted tree at each corner of the map, where a corner is a mutually incident pair of edges, vertex and face. The resulting map may either inherit the rooting of the smooth map or be rooted at a vertex of a tree. This is achieved by means of the factor $v z^{-1} \partial z / \partial v$, in which $v \partial z / \partial v$ is the generating series for doubly rooted plane planted trees. This construction has been observed earlier by Bender and Canfield [2].

A similar result may be obtained, of course, relating $M(u, x, y, z)$ to the genus arises for homeomorphically irreducible maps. Clearly, $M$ and $M_{\overline{\{1,2\}}}$ are obtainable, one from the other, by these constructions.

The next result, which concerns monopoles, is required in the determination of the genus series for dipoles. In this case, the integration must be carried out explicitly. The following results are needed for this purpose. Monopoles have been studied by Harer and Zagier [7] and by Jackson [10], using entirely different methods.

Hermite polynomials are othogonal with respect to the measure $e^{-x^{2}} d x$, so we give next properties to be used in completing the integration. Their standardisation is $\left[x^{n}\right] H_{n}(x)=2^{n}$, and they satisfy the orthogonality relation

$$
\int_{\mathbf{R}} H_{m}(x) H_{n}(x) e^{-x^{2}} d x=2^{n} n ! \sqrt{\pi} \delta_{m, n} .
$$

If $b_{k}(x)$ is a polynomial of degree at most $N-1$ in $x$ with $b_{j k}=\left[x^{j}\right] b_{k}$ for $k=0, \ldots, N-1$, and $V\left(x_{1}, \ldots, x_{N}\right)=\prod_{1 \leq j<k \leq N}\left(\lambda_{j}-\lambda_{k}\right)$, then

$$
\operatorname{det}\left[b_{k-1}\left(x_{j}\right)\right]_{N \times N}=V\left(x_{1}, \ldots, x_{N}\right) \operatorname{det}\left[b_{j k}\right]_{N \times N} .
$$

In particular, from the standardisation of the Hermite polynomials,

$$
\operatorname{det}\left[H_{k-1}\left(x_{j}\right)\right]_{N \times N}=\sqrt{2}^{N(N-1)} V\left(x_{1}, \ldots, x_{N}\right) .
$$

The use of (17) and the orthogonality of the Hermite polynomials is a familiar one in the context of the multivariate normal distribution [14].

Theorem 3.2. Let $m_{g}(n)$ be the number of monopoles of genus $g$ on $n$ edges.

$$
m_{g}(n)=\left[u^{n+1-2 g}\right] h^{\left[2^{n}\right]} \sum_{k=1}^{n+1}\left(\begin{array}{l}
u \\
k
\end{array}\right)\left(\begin{array}{c}
u \\
k-1
\end{array}\right) 2^{k-1} .
$$


Proof. From Theorem 1.1

$$
m_{g}(n)=\left[u^{2 g} x_{2 n} z^{n}\right] M\left(u^{2}, \mathbf{x}, 1, z\right)=\left[u^{n+1-2 g}\right] A_{[2 n]}(u) .
$$

From Corollary 2.7 and (17),

$$
\begin{aligned}
A_{[2 n]}(N)= & \frac{c_{N, n}}{2^{N^{2}-N}} \int_{\mathbb{R}^{N}} \operatorname{det}^{2}\left[H_{k-1}\left(\lambda_{j}\right)\right]_{N \times N} e^{-p_{2}} \sum_{q=1}^{N} \lambda_{q}^{2 n} d \lambda \\
= & \frac{c_{N, n}}{2^{N^{2}-N}} \sum_{q=1}^{N} \sum_{\sigma, \rho \in \mathfrak{G}_{N}} \operatorname{sgn}(\sigma \rho)\left(\prod_{\substack{1 \leq j \leq N \\
j \neq q}} 2^{\sigma(j)-1}(\sigma(j)-1) ! \sqrt{\pi} \delta_{\sigma(j)-1, \rho(j)-1}\right) \\
& \cdot \int_{\mathbb{R}} \lambda_{q}^{2 n} H_{\sigma(q)-1}\left(\lambda_{q}\right) H_{\rho(q)-1}\left(\lambda_{q}\right) e^{-\lambda_{q}^{2}} d \lambda_{q},
\end{aligned}
$$

by orthogonality. For nonzero contributions, $\sigma(j)=\rho(j)$ for all $j \in\{1, \ldots$, $N\}-\{q\}$. Thus $\sigma(q)=\rho(q)$ since $\sigma, \rho$ are bijective, whence $\sigma=\rho$. It follows from (15) and (17) that

$$
\begin{aligned}
A_{[2 n]}(N) & =\frac{2^{n}}{\sqrt{\pi} N !} \sum_{q=1}^{N} \sum_{r=0}^{N-1} \sum_{\substack{\sigma \in \mathfrak{G}_{N} \\
\sigma(q)=r+1}} \int_{\mathbb{R}} x^{2 n} \frac{H_{r}^{2}(x)}{2^{r} r !} e^{-x^{2}} d x \\
& =\frac{2^{n}}{\sqrt{\pi}} \int_{\mathbb{R}} x^{2 n} \sum_{r=0}^{N-1} \frac{H_{r}^{2}(x)}{2^{r} r !} e^{-x^{2}} d x .
\end{aligned}
$$

But (see, for example, [15])

$$
\begin{aligned}
\frac{1}{2^{r} r !^{2}} H_{r}^{2}(x) & =\sum_{k=0}^{r} \frac{H_{2 k}(x)}{2^{k} k !^{2}(r-k) !}, \\
\int_{\mathbb{R}} x^{2 n} H_{2 k} e^{-x^{2}} d x & = \begin{cases}2^{-2(n-k)} \frac{(2 n) !}{(n-k) !} \sqrt{\pi} & \text { if } n \geq k, \\
0 & \text { otherwise } .\end{cases}
\end{aligned}
$$

Thus

$$
A_{[2 n]}(N)=\frac{(2 n) !}{2^{n} n !} \sum_{k=1}^{n+1}\left(\begin{array}{l}
N \\
k
\end{array}\right)\left(\begin{array}{c}
n \\
k-1
\end{array}\right) 2^{k-1},
$$

assuming that $N>n$. This is a polynomial in $N$ and the result follows.

The remainder of this section is concerned with dipoles. In principle, the approach adopted here can be extended to vertex-regular maps in general. Two mappings, $\eta$ and $\varpi$, as required. The first [5] determines the linear coefficient in formal power series.

Proposition 3.3. Let $\mathbb{R}$ be a ring, let $\phi \in \mathbb{R}[[\mathbf{x}]]^{\mathfrak{G}}$, and let $h_{k}(\mathbf{x})$ be the complete symmetric function in $\mathbf{x}=\left(x_{1}, x_{2}, \ldots\right)$ of degree $k$. Let

$$
\eta: \mathbb{R}[[\mathbf{x}]]^{\mathfrak{G}} \rightarrow \mathbb{R}: h_{j} \mapsto x^{j} / j !,
$$

extended as a homomorphism to the whole of the ring. Then

$$
\left[x_{1} \cdots x_{n}\right] \phi=\left[x^{n} / n !\right] \eta \phi .
$$


Proof. The result is unaffected by restricting to the quotient ring

$$
\mathbb{R}\left[\left[x_{1}, \ldots, x_{n}\right]\right]^{\mathbb{B}} /\left\{x_{i}^{2}=0, i=1, \ldots, n ; x_{n+1}=\cdots=0\right\},
$$

so $h_{k}=\left[t^{k}\right] \prod_{j \geq 1}\left(1-t x_{j}\right)^{-1}=\left[t^{k}\right] e^{t\left(x_{1}+\cdots+x_{n}\right)}=\frac{1}{k !}\left(x_{1}+\cdots+x_{n}\right)^{k}$. Thus

$$
\left[x_{1} \cdots x_{n}\right] h_{1}^{k_{1}} \cdots h_{n}^{k_{n}}=\left[x_{1} \cdots x_{n}\right] \frac{\left(x_{1}+\cdots+x_{n}\right)^{n}}{1 ! k_{1} \cdots n !^{k_{n}}}=\left[\frac{x^{n}}{n !}\right]\left(\frac{x}{1 !}\right)^{k_{1}} \cdots\left(\frac{x^{n}}{n !}\right)^{k_{n}} .
$$

The result follows by linearity.

The second mapping is needed in the construction of the diagonal of a twovariable symmetric function, which is uniquely expressible in terms of two algebraically independent symmetric invariants, namely $y+z$ and $y z$.

Proposition 3.4. Let

$$
\varpi: \mathbb{Q}[u][[y, z]]^{\mathfrak{G}} \rightarrow \mathbb{Q}[[u, t]]:(y, z)^{m}(y z)^{k} \mapsto\left(\begin{array}{c}
m \\
\left\lfloor\frac{1}{2} m\right\rfloor
\end{array}\right) t^{\left\lfloor\frac{1}{2} m\right\rfloor+k} \delta_{0, m \bmod 2},
$$

extended linearly to the whole of $\mathbb{Q}[u][[y, z]]^{\mathfrak{B}}$. Let $f \in \mathbb{Q}[u][[y, z]]^{\mathfrak{B}}$. Then

$$
\begin{aligned}
& \text { (1) }\left[y^{m} z^{m}\right] f=\left[t^{m}\right] \varpi f, \\
& \text { (2) } \varpi f(y z, y+z)=\varpi f(t, y+z) \text {. }
\end{aligned}
$$

That is, $\varpi f$ is the $\{y, z\}$-diagonal of $f$, and $\varpi$ is a partial homomorphism. The next theorem is the main result of this section, and it gives an explicit expression for the genus series for dipoles.

Theorem 3.5. The number $d_{g}(n)$ of dipoles of genus $g$ on $n$ edges is

$$
\begin{array}{r}
{\left[\frac{u^{n-2 g}}{(n-1) !}\right] \sum_{j=0}^{\left\lfloor\frac{1}{2}(n-1)\right\rfloor}\left(\begin{array}{c}
2 j \\
j
\end{array}\right) \sum_{r=0}^{n-2 j-1}\left(\begin{array}{c}
n-2 j-1 \\
r
\end{array}\right)} \\
\times\left(\begin{array}{c}
u+j+r \\
n
\end{array}\right) \sum_{k=0}^{\left\lfloor\frac{1}{2}(n-2 j-1)\right\rfloor} \frac{1}{4^{k}}\left(\begin{array}{c}
2 k \\
k
\end{array}\right)\left(\begin{array}{c}
n \\
2 k
\end{array}\right) .
\end{array}
$$

Proof. From Theorem 1.1

$$
d_{g}(n)=\left[u^{2 g} x_{n}^{2} z^{n}\right] M\left(u^{2}, \mathbf{x}, 1, z\right)=\frac{1}{n}\left[u^{n-2 g}\right]\left(A_{\left[n^{2}\right]}(u)-A_{[n]}^{2}(u)\right) .
$$

But, from (17) and Corollary 2.7, $A_{\left[n^{2}\right]}(N)=A_{[2 n]}(N)+J_{n, N}$ where

$$
\begin{aligned}
J_{n, N}= & c_{N, n} \int_{\mathbb{R}^{N}} V^{2}(\lambda) e^{-p_{2}} \sum_{1 \leq q \neq q^{\prime} \leq N} \lambda_{q}^{n} \lambda_{q^{\prime}}^{n} d \lambda \\
= & \frac{c_{N, n}}{2^{N^{2} N n}} \int_{\mathbb{R}^{N}} \operatorname{det} 2\left[H_{k-1}\left(\lambda_{j}\right)\right]_{N \times N} e^{-p_{2}} \sum_{1 \leq q \neq q^{\prime} \leq N} \lambda_{q}^{n} \lambda_{q^{\prime}}^{n} d \lambda \\
= & \frac{c_{N, n}}{2^{N^{2}-N}} \sum_{\substack{1 \leq q, q^{\prime} \leq N \\
q \neq q^{\prime}}} \sum_{\sigma, \rho \in \mathfrak{B}_{N}} \operatorname{sgn}(\sigma \rho) \\
& \cdot\left(\prod_{\substack{1 \leq j \leq N \\
j \notin\left\{q, q^{\prime}\right\}}} 2^{\sigma(j)-1}(\sigma(j)-1) ! \sqrt{\pi} \delta_{\sigma(j)-1, \rho(j)-1}\right) \\
& \cdot \int_{\mathbf{R}} \lambda_{q}^{n} H_{\sigma(q)-1}\left(\lambda_{q}\right) H_{\rho(q)-1}\left(\lambda_{q}\right) e^{-\lambda_{q}^{2}} \lambda_{q^{\prime}}^{n} H_{\sigma\left(q^{\prime}\right)-1}\left(\lambda_{q^{\prime}}\right) H_{\rho\left(q^{\prime}\right)-1}\left(\lambda_{q^{\prime}}\right) e^{-\lambda_{q^{\prime}}^{2} d \lambda_{q} d \lambda_{q^{\prime}} .}
\end{aligned}
$$


For nonzero contributions we require that $\sigma(j)=\rho(j)$ for $j \in\{1, \ldots, N\}-$ $\left\{q, q^{\prime}\right\}$. Let $\sigma(q)=r, \sigma\left(q^{\prime}\right)=r^{\prime}$, where $r \neq r^{\prime}$, and $r, r^{\prime} \in\{1, \ldots, N\}$. There are therefore two choices for $\rho$, corresponding to $\rho(q)=r, \rho\left(q^{\prime}\right)=r^{\prime}$, and $\rho(q)=r^{\prime}, \rho\left(q^{\prime}\right)=r$. Let $\operatorname{Bij}\left(N, q, q^{\prime}, r, r^{\prime}\right)$ be the set of all bijections from $\{1, \ldots, N\}-\left\{q, q^{\prime}\right\}$ to $\{1, \ldots, N\}-\left\{r, r^{\prime}\right\}$. Then,

$$
\begin{aligned}
J_{n, N}= & \frac{2^{n}}{\pi N !} \sum_{\substack{1 \leq q, q^{\prime} \leq N \\
q \neq q^{\prime}}} \sum_{\substack{1 \leq r, r \leq N \\
r \neq r^{\prime}}} \frac{1}{2^{r-1}(r-1) !} \frac{1}{2^{r^{\prime}-1}\left(r^{\prime}-1\right) !} \sum_{\theta \in \operatorname{Bij}\left(N, q, q^{\prime}, r, r^{\prime}\right)} \\
& \cdot\left(\int_{\mathbb{R}} \lambda_{q}^{n} H_{r-1}^{2}\left(\lambda_{q}\right) e^{-\lambda_{q}^{2}} d \lambda_{q} \int_{\mathbb{R}} \lambda_{q^{\prime}}^{n} H_{r^{\prime}-1}^{2}\left(\lambda_{q^{\prime}}\right) e^{-\lambda_{q^{\prime}}^{2} d \lambda_{q^{\prime}}}\right. \\
& \left.-\int_{\mathbb{R}^{2}} \lambda_{q}^{n} H_{r-1}\left(\lambda_{q}\right) H_{r^{\prime}-1}\left(\lambda_{q}\right) e^{-\lambda_{q}^{2}} \lambda_{q^{\prime}}^{n} H_{r^{\prime}-1}\left(\lambda_{q^{\prime}}\right) H_{r-1}\left(\lambda_{q^{\prime}}\right) e^{-\lambda_{q^{\prime}}^{2} d \lambda_{q} d \lambda_{q^{\prime}}}\right) \\
= & D_{n, N}-C_{n, N}
\end{aligned}
$$

where

$$
\begin{aligned}
& D_{n, N}=\frac{2^{n}}{\pi}\left(\int_{\mathbb{R}} x^{n}\left(\sum_{r=0}^{N-1} \frac{H_{r}^{2}(x)}{2^{r} r !}\right) e^{-x^{2}} d x\right)^{2}=A_{[n]}^{2}(N) \quad \text { from (18), } \\
& C_{n, N}=\frac{2^{n}}{\pi} \int_{\mathbb{R}^{2}} x^{n} y^{n}\left(\sum_{r=0}^{N-1} \frac{H_{r}(x) H_{r}(y)}{2^{r} r !}\right)^{2} e^{-x^{2}-y^{2}} d x d y .
\end{aligned}
$$

Using Mehler's theorem [15], which states that

$$
\sum_{k \geq 0} H_{k}(a) H_{k}(b) \frac{z^{k}}{2^{k} k !}=\frac{1}{\sqrt{1-z^{2}}} \exp \left(\frac{2 a b z-\left(a^{2}+b^{2}\right) z^{2}}{1-z^{2}}\right),
$$

we have

$$
\begin{aligned}
C_{n, N}= & \frac{1}{\pi}\left[(y z)^{N} \frac{u^{n}}{n !}\right] \frac{y}{1-y} \frac{z}{1-z}\left(\frac{1}{\left(1-y^{2}\right)\left(1-z^{2}\right)}\right)^{1 / 2} \\
& \cdot \int_{\mathbb{R}^{2}} e^{-\left(A a^{2}-2 H a b+A b^{2}\right)} d a d b
\end{aligned}
$$

where

$$
A=1+\frac{y^{2}}{1-y^{2}}+\frac{z^{2}}{1-z^{2}}, \quad H=u+\frac{y}{1-y^{2}}+\frac{z}{1-z^{2}},
$$

so, by diagonalizing the quadratic form and integrating,

$$
\begin{aligned}
C_{n, N} & =\left[t^{N} \frac{u^{n}}{n !}\right] \varpi \frac{y}{1-y} \frac{z}{1-z}\left(\frac{1}{\left(1-y^{2}\right)\left(1-z^{2}\right)\left(A^{2}-H^{2}\right)}\right)^{1 / 2} \\
& =\left[t^{N-1} \frac{u^{n}}{n !}\right] \varpi G,
\end{aligned}
$$

where $G=(1-\alpha+w)^{-1}\left\{(1-w-u \alpha)^{2}-u^{2}(1+w)^{2}\right\}^{-1 / 2}$, with $\alpha=y+z$ and $w=y z$. Combining these results gives

$$
A_{\left[n^{2}\right]}(N)-A_{[n]}^{2}(N)=A_{[2 n]}(N)-\left[t^{N-1} u^{n} / n !\right] \varpi G
$$


where, from Proposition 3.4,

$$
\left[u^{n}\right] \varpi G=\sum_{k=0}^{\left\lfloor\frac{1}{2} n\right\rfloor}(-1)^{k}\left(\begin{array}{c}
-\frac{1}{2} \\
k
\end{array}\right)\left(\begin{array}{c}
n \\
2 k
\end{array}\right) \frac{(1+t)^{2 k}}{(1-t)^{n+1}} \varpi \frac{\alpha^{n-2 k}}{1-\alpha+t} .
$$

But for any nonnegative integer $p$,

$$
\varpi \frac{\alpha^{p}}{1-\alpha+t}=\frac{(1+t)^{p}}{1-t}-\sum_{j=0}^{\left\lfloor\frac{1}{2}(p-1)\right\rfloor}\left(\begin{array}{c}
2 j \\
j
\end{array}\right) t^{j}(1+t)^{p-1-2 j},
$$

by direct computation of the action of $\varpi$, so $\left[t^{N-1} u^{n} / n !\right] \varpi G=F_{n, N}-E_{n, N}$ where

$$
\begin{aligned}
F_{n, N} & =n !\left[t^{N-1}\right] \sum_{k=0}^{\left\lfloor\frac{1}{2} n\right\rfloor}(-1)^{k}\left(\begin{array}{c}
-\frac{1}{2} \\
k
\end{array}\right)\left(\begin{array}{c}
n \\
2 k
\end{array}\right) \frac{(1+t)^{n}}{(1-t)^{n+2}} \\
& =\frac{(2 n) !}{2^{n} n !} \sum_{k=0}^{n}\left(\begin{array}{l}
n \\
k
\end{array}\right)\left(\begin{array}{c}
N+k \\
n+1
\end{array}\right) \\
& =\frac{(2 n) !}{2^{n} n !}\left[t^{n+1}\right](1+t)^{N}(2+t)^{n}=A_{[2 n]}(N)
\end{aligned}
$$

from (19), and

$$
E_{n, N}=n !\left[t^{N-1}\right] \sum_{k=0}^{\left\lfloor\frac{1}{2} n\right\rfloor}(-1)^{k}\left(\begin{array}{c}
-\frac{1}{2} \\
k
\end{array}\right)\left(\begin{array}{c}
n \\
2 k
\end{array}\right) \sum_{j=0}^{\left\lfloor\frac{1}{2}(n-2 k-1)\right\rfloor}\left(\begin{array}{c}
2 j \\
j
\end{array}\right) t^{j} \frac{(1+t)^{n-2 j-1}}{(1-t)^{n+1}}
$$

Thus $A_{\left[n^{2}\right]}(N)-A_{[n]}^{2}(N)=E_{n, N}$ and $E_{n, N}$ is exhibited as a polynomial in $N$, and the result now follows from (20).

The expression given in Theorem 3.5 conceals the parity of the genus series for dipoles. A parity respecting form can be derived by changing basis to $\left\{(u-i)_{2 i-1}: i \geq 0\right\}$ consisting of odd series. The proof is given elsewhere [1], but the result for $n$ odd is included below for completeness.

Theorem 3.6. $\left(\begin{array}{c}2 m \\ m\end{array}\right)^{-1} d_{g}(2 m+1)$ is equal to

$$
\left[\frac{u^{2 m-2 g+1}}{(2 m+1) !}\right] \sum_{i=0}^{m} \frac{(u-i)_{2 i+1}}{(2 i+1) !} 4^{i-m}\left(\begin{array}{c}
m \\
i
\end{array}\right) \sum_{k=0}^{m-i} \frac{(-1)^{k}}{2 k+1}\left(\begin{array}{c}
m-i \\
k
\end{array}\right)\left(\begin{array}{c}
2 m-k \\
m
\end{array}\right) .
$$

The basis is well adapted to hypergeometric series, and can be used to facilitate the coefficient extraction.

\section{OTHER CONSEQUENCES OF THE INTEGRAL REPRESENTATION}

The two results of this section can be regarded as consequences of arguments based on maps. A further example is given in [6].

The first result concerns character sums. Let $\chi_{\phi}^{\theta}$ denote the value of the character of the irreducible representation of $\mathfrak{G}_{n}$ indexed by $\theta$ on any element of the conjugacy class, $\mathscr{C}_{\phi}$, indexed by $\phi$, and let $f^{\theta}$ denote the degree of the representation. For $\mu \vdash n$, let $s_{\mu}$ denote the Schur symmetric function indexed by $\mu$. Let $H_{\theta}(x)=\prod_{1 \leq i \leq l(\theta)}(x-i+1)^{\left(\theta_{i}\right)}$, where $(x)^{(n)}=x(x+1) \cdots(x+n-1)$. 
Theorem 4.1. Let $\nu \vdash 2 n$, and let $p_{\nu}$ denote $p_{\nu}\left(\lambda_{1}, \ldots, \lambda_{N}\right)$, where $N$ is a positive integer. Then

$$
H_{\theta}(N) \chi_{\left[2^{n}\right]}^{\theta}=\frac{2^{n} n !}{(2 \pi)^{\frac{1}{2} N}} \frac{1}{\prod_{j=1}^{N} j !} \int_{\mathbb{R}^{N}} V^{2}(\lambda) e^{-1 / 2 p_{2}} s_{\theta} d \lambda .
$$

Proof. Let $\nu$ be an arbitrary but fixed element of $\mathscr{C}_{\alpha}$, where $\alpha \vdash 2 n$. Let $\mathbb{K}_{\alpha}=\sum_{g \in \mathscr{C}_{\alpha}} g$. We now work in the centre of $\mathbb{C G}_{2 n}$. The number of ways of expressing an arbitrary element $c \in \mathscr{C}_{\phi}$ as $c=\nu b$ with $(\nu, b)=\mathscr{C}_{\alpha} \times \mathscr{C}_{\left[2^{n}\right]}$ is $\left(h^{\phi} / h^{\alpha}\right)\left[\mathbb{K}_{\phi}\right] \mathbb{K}_{\alpha} \mathbb{K}_{\left[2^{n}\right]}$, so

$$
\sum_{\pi \in \nu \mathscr{E}_{\left[2^{n}\right]}} N^{\kappa(\pi)}=\frac{1}{h^{\alpha}} \sum_{\phi \vdash 2 n} N^{l(\phi)} h^{\phi}\left[\mathbb{K}_{\phi}\right] \mathbb{K}_{\alpha} \mathbb{K}_{\left[2^{n}\right]} .
$$

But, using the orthogonal idempotents spanning the centre,

$$
\left[\mathbb{K}_{\phi}\right] \mathbb{K}_{\alpha} \mathbb{K}_{\left[2^{n}\right]}=\frac{1}{(2 n) !} h^{\alpha} h^{\left[2^{n}\right]} \sum_{\theta \vdash 2 n} \frac{1}{f^{\theta}} \chi_{\phi}^{\theta} \chi_{\alpha}^{\theta} \chi_{\left[2^{n}\right]}^{\theta},
$$

and (see, for example, [13]) $\sum_{\phi \vdash 2 n} h^{\phi} \chi_{\phi}^{\theta} x^{l(\phi)}=f^{\theta} H_{\theta}(x)$, so

$$
\sum_{\pi \in \nu \mathscr{E}_{\left[2^{n}\right]}} N^{\kappa(\pi)}=\frac{h^{\left[2^{n}\right]}}{(2 n) !} \sum_{\theta \vdash 2 n} H_{\theta}(N) \chi_{\alpha}^{\theta} \chi_{\left[2^{n}\right]}^{\theta} .
$$

Thus, from (1) and Theorem 2.6

$$
\begin{aligned}
\sum_{\theta \vdash 2 n} H_{\theta}(N) \chi_{\alpha}^{\theta} \theta_{\left[2^{n}\right]}^{\theta} & =2^{n} n !\left\langle\prod_{k \geq 1}\left(\operatorname{tr} \mathbf{M}^{k}\right)^{a_{k}}\right\rangle \\
& =\frac{2^{n} n !}{\left(2^{N} \pi^{N^{2}}\right)^{1 / 2}} \int_{\mathscr{V}_{N}} \prod_{k \geq 1}\left(\operatorname{tr} \mathbf{M}^{k}\right)^{a_{k}} e^{-\frac{1}{2} \operatorname{tr} \mathbf{M}^{2}} d \mathbf{M} \\
& =\frac{2^{n} n !}{(2 \pi)^{\frac{1}{2} N}} \frac{1}{\prod_{j=1}^{N} j !} \int_{\mathbb{R}^{N}} V^{2}(\lambda) e^{-\frac{1}{2} p_{2}} p_{\alpha} d \lambda,
\end{aligned}
$$

from (14), and the result follows by the orthogonality of the characters.

\section{Corollary 4.2.}

$$
\sum_{\theta \vdash 2 n} H_{\theta}(x)\left(\chi_{\left[2^{n}\right]}^{\theta}\right)^{2}=2^{n} n ! x^{2}\left(x^{2}+2\right)\left(x^{2}+4\right) \cdots\left(x^{2}+2 n-2\right) .
$$

Proof. Let $N$ be a positive integer, and let $t_{n}=\sum_{\theta \vdash 2 n} H_{\theta}(N)\left(\chi_{\left[2^{n}\right]}^{\theta}\right)^{2}$. Let $u$ be an indeterminate. Then, from (21)

$$
\sum_{n \geq 0}(-1)^{n} \frac{t_{n}}{4^{n} n !^{2}} u^{n}=\frac{1}{\sqrt{2^{N} \pi^{N^{2}}}} \int_{\mathscr{V}} e^{-\frac{1}{2}(1+u) \operatorname{tr} \mathbf{M}^{2}} d \mathbf{M}
$$

so, from (13), $t_{n}=(-4)^{n} n !^{2}\left[u^{n}\right](1+u)^{-\frac{1}{2} N^{2}}$, a polynomial in $N$, and the result follows.

The second result concerns a factorisation of the integrals which can be deduced from a property of quadrangulations implied by (2). 
Theorem 4.3. Let $v$ be an indeterminate. Then

$$
\left\langle e^{\frac{1}{2} v \operatorname{tr} \mathbf{M}^{4}}\right\rangle_{\mathscr{V}_{2 N}}=\left\langle\left|\mathbf{I}_{N}-2 \sqrt{v} \mathbf{M}\right|^{-\left(N+\frac{1}{2}\right)}\right\rangle_{\mathscr{V}_{N}} \cdot\left\langle\left|\mathbf{I}_{N}-2 \sqrt{v} \mathbf{M}\right|^{-\left(N-\frac{1}{2}\right)}\right\rangle_{\mathscr{V}_{N}}
$$

Proof. From Theorem 1.1, $M\left(u^{2}, \mathbf{x}, y, z\right)=\Omega_{u} \log S(\mathbf{x}, y, z)$ where

$$
S(\mathbf{x}, y, z)=\sum_{n \geq 0} \frac{2^{n} z^{n}}{(2 n) !} \sum_{\nu \vdash 2 n} h^{\nu} A_{\nu}(y) \mathbf{x}^{m(\nu)} \in \mathbb{Q}[\mathbf{x}, y][[z]] .
$$

Thus, $M\left(u^{2}, x, \mathbf{y}, z\right)=\Omega_{u} \log R(x, \mathbf{y}, z)$ where

$$
R(x, \mathbf{y}, z)=\sum_{n \geq 0} \frac{2^{n} z^{n}}{(2 n) !} \sum_{\phi \vdash 2 n} h^{\phi} A_{\phi}(x) \mathbf{y}^{m(\phi)} \in \mathbb{Q}[x, \mathbf{y}][[z]],
$$

in which $\mathbf{y}=\left(y_{1}, y_{2}, \ldots\right)$ and $y_{j}$ marks faces bounded by $j$ edges. Let $R(x, y, z)$ denote $R(x, \mathbf{y}, z)$ denote $R(x, \mathbf{y}, z)$ at $y_{1}=y_{2}=\cdots=y$, and let $R_{4}(x, y, z)$ at $y_{k}=y \delta_{k, 4}$ for $k \geq 1$. But from (Theorem 5.1 [11])

$$
R_{4}(x, y, z)=R\left(\frac{1}{2} x, \frac{1}{2}(x+1), 4 z^{2} y\right) R\left(\frac{1}{2}(x-1), \frac{1}{2} x, 4 z^{2} y\right) .
$$

Let $S(x, y, z)$ denote $S(\mathbf{x}, y, z)$ at $x_{1}=x_{2}=\cdots=x$, and let $S_{4}(x, y, z)$ denote $S(\mathbf{x}, y, z)$ at $x_{k}=x \delta_{k, 4}$ for $k \geq 1$. Then, from (22)

$$
S_{4}(x, y, z)=S\left(\frac{1}{2} y, \frac{1}{2}(y+1), 4 z^{2} x\right) S\left(\frac{1}{2}(y-1), \frac{1}{2} y, 4 z^{2} x\right) .
$$

By duality, and setting $y=N$,

$$
S_{4}(x, 2 N, z)=S\left(N+\frac{1}{2}, N, 4 z^{2} x\right) S\left(N-\frac{1}{2}, N, 4 z^{2} x\right) .
$$

The result follows from Theorem 2.6 after replacing $2 z^{2} x$ by $v$.

Equivalently, from Corollary 2.7,

$$
\begin{aligned}
& \int_{\mathbb{R}^{2 N}} V^{2}(\lambda) e^{-\frac{1}{2} p_{2}+\frac{1}{2} v p_{4}} d \lambda \\
&=\pi^{N^{2}} \int_{\mathbb{R}^{N}} V^{2}(\lambda) e^{-\frac{1}{2} p_{2}} \prod_{j=1}^{N}\left(1-2 \sqrt{v} \lambda_{j}\right)^{-\left(N+\frac{1}{2}\right)} d \lambda \\
& \cdot \int_{\mathbb{R}^{N}} V^{2}(\lambda) e^{-\frac{1}{2} p_{2}} \prod_{j=1}^{N}\left(1-2 \sqrt{v} \lambda_{j}\right)^{-\left(N-\frac{1}{2}\right)} d \lambda .
\end{aligned}
$$

It would be helpful to have a more direct proof of Theorem 4.3 to see whether such a factorisation also holds for real Hermitian matrices.

\section{EXTENSION OF THE LINEAR FUNCTIONAL}

An argument analogous to the one given in the proof of Lemma 2.3 gives a summation theorem for the general form of the linear functional, and thence an expression for products with the conjugacy class $\mathscr{C}_{\left[K^{n}\right]}$. Let $i_{1}, \ldots, i_{K n} \leq N$ be positive integers and let $\mathbf{x}=\left(x_{1}, \ldots, x_{N}\right)$. Then

$$
\sum_{\rho \in \mathscr{E}_{\left[K^{n}\right]}} \prod_{j=1}^{K^{n}}\left\langle x_{i_{j}} x_{i_{\rho(j)}} \cdots x_{i_{\rho^{K-1}(j)}}\right\rangle_{K, \mathbf{d}}^{1 / K}=\left\langle x_{i_{1}} \cdots x_{i_{N}}\right\rangle_{K, \mathbf{d}}
$$


If $g_{k}(\mathbf{x})$ is a homogeneous linear polynomial in $\mathbf{x}$, for $k=1, \ldots, N$, then

$$
\sum_{\rho \in \mathscr{C}_{\left[K^{n}\right]}} \prod_{j=1}^{K^{n}}\left\langle g_{i_{j}}(\mathbf{x}) g_{i_{\rho(j)}}(\mathbf{x}) \cdots g_{i_{\rho} K-1_{(j)}}(\mathbf{x})\right\rangle_{K, \mathbf{d}}^{1 / K}=\left\langle g_{i_{1}}(\mathbf{x}) \cdots g_{i_{N}}(\mathbf{x})\right\rangle_{K, \mathbf{d}}
$$

Lemma 2.3 corresponds to the case $K=2$. Thus, for arbitrary homogeneous polynomials $f_{j k}$ in the elements of $\mathbf{A}$, and for any $\nu \in \mathfrak{G}_{K n}$,

$$
\begin{gathered}
\sum_{\rho \in \mathscr{E}_{\left[K^{n}\right]}} \prod_{j=1}^{K n}\left\langle f_{i_{j}, i_{\nu(j)}(\mathbf{A}) f_{i \rho(j)}, i_{\nu \rho(j)}}(\mathbf{A}) \cdots f_{i_{\rho^{K-1}(j)}}(\mathbf{A})\right\rangle_{K, \mathbf{D}}^{1 / K} \\
=\left\langle f_{i_{1}, i_{\nu(1)}}(\mathbf{A}) \cdots f_{i_{K n}, i_{\nu(K n)}}(\mathbf{A})\right\rangle_{K, \mathbf{D}}
\end{gathered}
$$

It follows that

$$
\begin{aligned}
& \sum_{\pi \in \mathscr{C}_{\left.\nu_{[} K^{n}\right]}} N^{\kappa(\pi)}=\sum_{1 \leq i_{1}, \ldots, i_{K n} \leq N} \sum_{\rho \in \mathscr{C}_{\left[K^{n}\right]}} \prod_{j=1}^{K n} \delta_{i_{j}, i_{\nu \rho(j)}} \cdots \delta_{i_{\rho}^{K-1}(j)}, i_{\nu \rho} K_{(j)} \\
&=\sum_{1 \leq i_{1}, \ldots, i_{K n} \leq N} \sum_{\rho \in \mathscr{C}_{\left[K^{n}\right]}} \prod_{j=1}^{K n} \psi\left(\left(i_{j}, i_{\rho(j)}, \ldots, i_{\rho^{K-1}(j)}\right),\right. \\
&\left.\left(i_{\nu(j)}, i_{\nu \rho(j)}, \ldots, i_{\nu^{K-1}(j)}\right)\right)
\end{aligned}
$$

where the appropriate connector function for the conjugacy class $\mathscr{C}_{\left[K^{n}\right]}$ is

$$
\psi\left(\left(q_{1}, \ldots, q_{K}\right),\left(r_{1}, \ldots, r_{K}\right)\right)=\delta_{q_{1}, r_{2}} \delta_{q_{2}, r_{3}} \cdots \delta_{q_{K-1}, r_{K}} \delta_{q_{K}, r_{1}} .
$$

To complete the argument it is of course necessary to supply an integral representation, with multiplicative kernel, for this connector function. We do not pursue this direction of enquiry further here.

\section{ACKNOWLEDGMENTS}

This work was carried out while the author was a Visiting Scholar in the Department of Mathematics at MIT from September 1990 to March 1991, and was completed during a visit to the Department of Pure Mathematics and Mathematical Statistics, University of Cambridge, in May and June 1992. The author thanks Persi Diaconis, Malcolm Perry, Richard Stanley and Terry Visentin for helpful conversations.

\section{REFERENCES}

1. G. E. Andrews, D. M. Jackson, and T. I. Visentin, A hypergeometric analysis of the genus series for a class of 2-cell embeddings in orientable surfaces, SIAM. J. Math. Anal. (to appear).

2. E. A. Bender and E. R. Canfield, The asymptotic number of rooted maps on a surface, J. Combin. Theory Ser. A 43 (1986), 244-257.

3. D. Bessis, C. Itzykson, and J. B. Zuber, Quantum field theory techniques in graphical enumeration, Adv. in Appl. Math. 1 (1980), 109-157.

4. F. J. Dyson, Statistical theory of energy levels of complex systems. I, J. Math. Phys. 3 (1962), 140-156.

5. I. P. Goulden and D. M. Jackson, Combinatorial enumeration, Wiley, New York, 1983.

6. Symmetric functions and Macdonald's result for top connexion coefficients in the symmetric group, J. Algebra (to appear). 
7. J. Harer and D. Zagier, The Euler characteristic of the moduli space of curves, Invent. Math. 85 (1986), 457-485.

8. G. 't Hooft, A planar diagram theory for string interactions, Nuclear Phys. B 72 (1974), 461-473.

9. C. Itzykson and J-M. Drouffe, Statistical field theory, Vol. 2, Cambridge Univ. Press, Cambridge, 1989.

10. D. M. Jackson, Counting cycles in permutations by group characters, with an application to a topological problem, Trans. Amer. Math. Soc. 299 (1987), 785-801.

11. D. M. Jackson and T. I. Visentin, $A$ character theoretic approach to embeddings of rooted maps in an orientable surface of given genus, Trans. Amer. Math. Soc. 322 (1990), 343-363.

12. J. C. Kluyver, A local probability problem, Nederl. Akad. Wetensch. Proc. Ser. B 8 (1906), 341-350.

13. I. G. Macdonald, Symmetric functions and Hall polynomials, Clarendon Press, Oxford, 1979.

14. M. L. Mehta, Random matrices, Academic Press, London, 1967.

15. G. Szegö, Orthogonal polynomials, Colloq. Publ., vol. 23, Amer. Math. Soc., Providence, RI, 1939.

16. H. Weyl, The classical groups, 2nd ed., Princeton Univ. Press, Princeton, NJ, 1946.

DEPARTMENT OF COMBINATORICS AND OPTIMIZATION, UNIVERSITY OF WATERLOO, WATERLOO, ONTARIO, CANADa

E-mail address: dmjackson@dragon.uwaterloo.ca 